\title{
s.s. \\ General Analytical Engine for Touch Based Systems (GAETS)
}

\author{
Preeti Gupta, Bhavana Thakur, Urvashi Bhatia, Harshada Wadekar \& S. B. Thakare \\ University of Pune, Pune, India
}

\begin{abstract}
Touch based systems are increasingly used to showcase products in the current markets. However, a robust system is needed to analyse the response and activities. We introduce an interactive general analytical engine (GAETS) designed to facilitate the creation of descriptive analysis done for touch based hardware. We intend to do this by keeping a tab on the number and variety of people who are using the hardware and analyse their actions based on what is displayed on the screen. This project will be implemented in three phases. The first phase will include image capturing, face recognition and image retrieval. Secondly, image processing will be done on the face detected (for detecting gender and age group) as well as on the icons the user accessed. Finally, a detailed analysis of the data gathered will be done and reports will be demonstrated visually on a dash board. This system will help the market analysts to know current market trends and sales patterns classified on the basis of gender and age group. The system would be modular in design to allow customization while retaining a flexible and stable structure.
\end{abstract}

Keywords - Analytical engine, gender detection, image processing.

\section{INTRODUCTION}

Analytics involve finding out meaningful patterns in the available set of data. The 'General Analytical Engine for Touch Based system' will generate the statistics by analysing the actions of a person to a particular product on the touch based kiosk depending on its age and gender. Strong popularity of touch screen kiosks suggests more businesses must adapt by integrating the technology into their service delivery. Kiosk is commonly used to give key information or collect user feedback and other data.

However, a robust system is needed to analyse the response and activities. We introduce an interactive general analytical engine (GAETS) designed to facilitate the creation of descriptive analysis done for touch based hardware. We intend to do this by keeping a tab on the number and variety of people who are using the hardware and analyse their actions based on what is displayed on the screen. This system will help the market analysts to know current market trends and sales patterns classified on the basis of gender and age group. The system would be modular in design to allow customization while retaining a flexible and stable structure.

\section{PREVIOUS WORK}

Earlier face recognition systems were mainly based on geometric facial features and template matching. In those works a face was characterized by a set of features such as mouth position, chin shape, nose width and length which are potentially insensitive to illumination conditions. The Principal Component Analysis (PCA) is one of the most successful techniques that have been used in image recognition and compression. PCA is a statistical method. The main idea of using PCA for face recognition is to express the large $1-\mathrm{D}$ vector of pixels constructed from 2-D facial image into the compact principal components of the feature space. The same principle is in effect used to recognize the gender of the test mage by evaluating the Euclidian distance of the test image from the images in the database. The proposed gender and face recognition technique using PCA is verified for both test images of a man and a woman. It was observed that if the number of images of a particular subject was more in the database, the gender recognition becomes even better.

\section{PROJECT SCOPE}

The project is an analytical engine for touch based hardware capable of capturing the images of people interacting with the system and at the same time classifying their image based on gender and age. The image captured will be first checked if it is already present in the database. If it is already present then his/her interactions with the hardware will be added to his existing account and if it is not present then a new account will be created and all his further interactions will be added to the newly created account.

The system will do the above mentioned activities for a specific period of time and will generate analytics in the form of visual representations based on the interactions of different categories of users. 


\section{OPERATING ENVIRONMENT}

Operating System: Windows XP, Windows 7

RAM $: 1 G B$

Hard disk $\quad: 4 G B$

Software Required: Visual studio 10, Open CV(library).

\section{IMPLEMENTATION DETAILS}

\section{Module1:}

Face detection-It is a technique to identify an object as a face and locate it in the input image. When the user gives his input to the system in the form of his first touch then the image of the user will be captured. The face detection algorithm will be applied to the captured image . The main aim of this module is to recognize facial features in the input image.

Module 2:

Face recognition-It is a technique to decide if the face is known or unknown based on the database of faces it uses to validate this input face. The image in which face is recognized is given as input to this module which checks if the face detected is the most recently used. If the image already exists then all his/her activities are added to his existing account but if the image does not exist then a new account is created.

\section{Module 3:}

Gender detection-This module aims to classify the input image based on gender. After the face recognition algorithm is applied to the algorithm then the image is classified on the basis of gender.

\section{Module 4:}

Approximate age group detection-The input image will be classified on the basis of three age groups i.e. children, young adult and senior adult.

Module 5:

Content based image retrieval-It is an application of computer vision techniques which addresses the problem of searching of digital images in large databases. This will be applied to the system when the image is to be searched in the database and also when the icons touched by the user are to be recognized.

\section{MATHEMATICAL MODEL}

The mathematical model is divided into two parts:
A: face detection
B: Face Recognition-Gender-Age classification

\section{A. FACE DETECTION:}

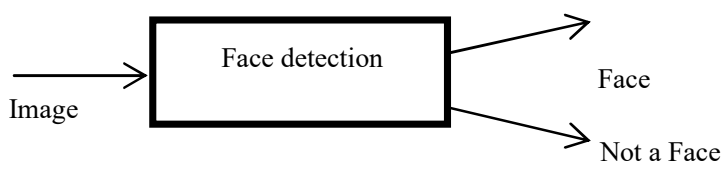

Let Rectangle_Feature_value be $f$,

$F=\sum$ (pixels in white area) $-\sum$ (pixels in shaded area)

If (f) is large it is face, i.e. if (f) $>$ threshold, then

Face;

Else

Non-face;

B. FACE RECOGNITION-GENDER-AGE CLASSIFICATION

1. Let $I_{1}, I_{2} \ldots, I_{M}$ be the training set of face images in the database. The average face is defined by:

$$
A=\frac{1}{M} \sum_{i=1}^{M} I_{i}
$$

2. Each face differs from the average face by the vector $Y_{i}=I_{1}-A$.

The covariance matrix $\mathrm{C}$ is obtained as

$$
C=\frac{1}{M} \sum_{i=1}^{M} Y_{i} \cdot Y_{i}^{T}
$$

3. The eigenvectors of the covariance matrix are computed and the $\mathrm{M}$ ' significant eigenvectors are chosen as those with the largest corresponding eigenvalues. From these eigenvectors, the weights for each image in the training set are computed as

$$
W_{i K}=E_{K}^{T} \cdot\left(I_{i}-A\right) \quad \forall i, k
$$

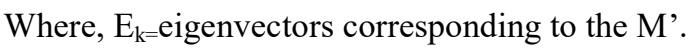

4. A test image $I_{\text {test }}$ is projected into face space by the following operation, derived from 3 above:

$$
W_{\text {test }}=E_{K}^{T} \cdot\left(I_{\text {test }}-A\right) \quad \forall K
$$

5. Each image in the training set is divided into $\mathrm{N}$ smaller images as follows:

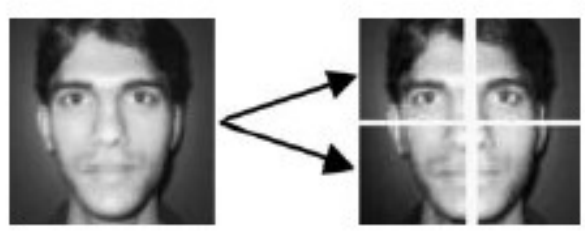

Hence the size of each sub-image will be $\mathrm{L}^{2} \sqrt{ } \mathrm{N}$.

These sub-images can be represented mathematically as

$$
I_{i j}(m, n)=I_{i}\left(\frac{L}{\sqrt{N}}(j-1)+m, \frac{L}{\sqrt{N}}(j-1)+n\right) \forall i, j
$$

Where, $1<\mathrm{i}<\mathrm{M}$, M being the number of images in the training set, $1<\mathrm{j}<\mathrm{N}, \mathrm{N}$ being the number of sub-images and $1<\mathrm{m}, \mathrm{n}<\mathrm{L} / \sqrt{ } \mathrm{N}$. 
6. The average image of all the training sub-images is computed as

$$
A=\frac{1}{M \cdot N} \sum_{i=1}^{M} \sum_{j=1}^{N} I_{i j}
$$

7. The next step is to normalize each training subimage by subtracting it from the mean as

$$
Y_{i j}=I_{i j}-A \quad \forall i, j
$$

8. From the normalized sub-images the covariance matrix is computed as

$$
C=\frac{1}{M . N} \sum_{i=1}^{M} \sum_{j=1}^{N} I_{i j} \cdot Y_{i j}^{T}
$$

9. Next we find the eigenvectors of $\mathrm{C}$ that are associated with the $M$ ' largest eigenvalues $\left(E_{1}, E_{2}\right.$, . $\ldots, \mathrm{E}_{\mathrm{M}}$ ) whose weights are :

$$
W_{p n K}=E_{K}^{T} \cdot\left(I_{p n j}-A\right) \quad \forall p, n, j, K
$$

10. Weights are also computed for the test sub-images using the eigenvectors as:

$$
W_{\text {test } j K}=E_{K}^{T} \cdot\left(I_{\text {test } j}-A\right) \quad \forall j, K
$$

11. Mean weight set of each class in the training set is computed from the weight sets of the class as shown below:

$$
T_{p j K}=\frac{1}{T} \sum_{r=1}^{M^{\prime}} \sum_{n=1}^{r} W_{p n j K} \quad \forall p, j
$$

12. Finally, the minimum distance is computed as shown below:

$$
\begin{gathered}
D_{p j}=\frac{1}{M^{\prime}} \sum_{K=1}^{M^{\prime}} \mid W_{\text {test } j K}-T_{p j K} \\
D_{p}=\frac{1}{N} \sum_{j=1}^{N} D_{p j}
\end{gathered}
$$

After successfully doing all the calculations, the image from the training set that has the minimum distance, is recognized and further calculations can be done on the same.
13. Gender Recognition is done using PCA using the same technique described above (in 1-12) except for the fact that the training set of images for gender detection is divided as Males and Females.

14. Age Recognition is done using PCA using the same technique described above (in 1-12) except for the fact that the training set of images for age detection is divided into age groups of:
i) Babies
ii) Teens
iii) Adults
iv) Old

\section{TECHNICAL SPECIFICATIONS}

A. Advantages

1. Touch based system hence no text bound interface, providing faster input leading to better customer service.

2. Shows analytics in a graphical format, thus provides easy way to interpret.

3. Provides easy approach to the administrator for updating the icons database.

4. Analytics generated will help the advertisers and market analysts to popularize a certain product in a particular age group of a specific gender.

B. Disadvantages

1. Requires initial investment for the hardware installment.

C. Applications:

1. Market Analysts:

The market analysts will use the analysis to know about the past and current market trends and predict future sales.

\section{Advertisement:}

The advertisers will specifically know which particular products have to advertise in which particular age-groups for males or females.

\section{HARDWARE DETAILS}

The hardware interface at the client side will include a Multi-touch MagixKiosk (by TouchMagix), while the one at the server side will have a laptop wherein the dashboard with the analytics will be displayed.

- The MagixKiosk ${ }^{\mathrm{TM}}$ can be adjusted easily into four form factors- Table, tilted kiosk/workstation, High Bar Table, or as a standing flat display.

- Super slim and robust design.

- $\quad 32 "$ HD high brightness LED display 
- Camera

\section{SOFTWARE DETAILS}

The project is designed specifically for Windows Operating system.

- $\quad$ Microsoft visual studio 10

- $\quad$ OpenCV (Library)

- $\mathrm{C}++$

\section{SYSTEM FEATURES}

Strong popularity of touch screen kiosks suggests more businesses must adapt by integrating the technology into their service delivery. We plan to do a detailed analysis of how a system is used by keeping a tab on the number and variety of people who are using the hardware and analyse their actions based on what is displayed on the screen. This system will help the market analysts to know current market trends and sales patterns classified on the basis of gender and age group.

The project will implement the image processing part in Visual Studio 10 with the help of OpenCV (Open Source Computer Vision Library). This library is cross platform and mainly focuses on real time image processing.

GAETS provides an export feature that allows the user to save contents of analysis results to an external location. This will help the advertisers and market analysts to popularize a certain product in a particular age group of a specific gender.

\section{SYSTEM ARCHITECTURE}

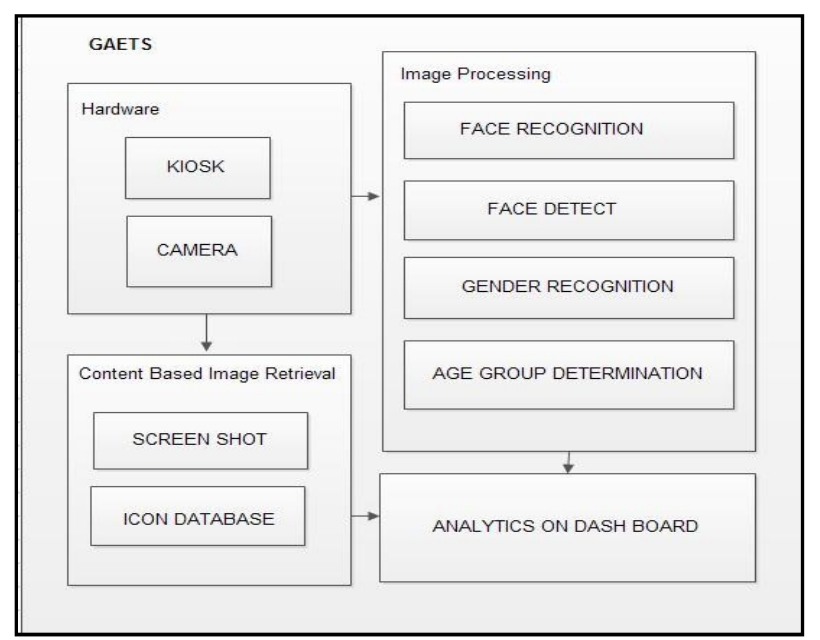

Fig. 1: System Architecture

\section{ACKNOWLEDGEMENT}

The initial development phase of this project is on its way to success due to the dedication, enthusiasm, support and cooperation of many people. We would like to acknowledge and express our gratitude to our staff members and well wishers. We have benefited greatly due to sincere efforts from our project guide and coordinators Mr. S. B. Thakare, Ms. V.M. Barkade and Dr. V.V. Phansalkar who gave us an opportunity to work on this topic and provided us the guidelines and standards for selecting project and helping us to choose our project topic appropriately.

We would also like to express our sincere thanks to Mr.Gaurav Shukla and Mr. Anup Agarwal of TouchMagix media pvt.ltd for providing us timely help and resources whenever required. We are also thankful to the whole team at TouchMagix media.pvt.ltd for their efforts to patiently listen and understand our project.

\section{REFERENCES}

\section{BOOKS:}

[1] Gonzalez Rafael C., Woods Richard E., Digital Image Processing, Publication Date: March 1992, Addison-Wesley, ISBN-10: 0201508036, ISBN13: 978-0201508031, Edition: $3^{\text {rd }}$.

\section{PAPERS}

[2] O. Ozbudak, M. Tukel, S. Seker, "Fast Gender Classification", Istanbul,Turkey, 2010, pp. 1-5.

[3] Jian-Gang Wang, Hee Lin Wang, Myint Ye and Wei-Yun Yau, "Real Time Gender recognition with Unaligned Face Images", Singapore, 2010, pp. 1-5

[4] Aly S. Abdelrahim, Mostafa A. Abdelrahman, Ali Mahmoud, and Aly A. Farag, "Image Retrieval Based on Content and Image Compression", USA, 2011, pp. 1-8

[5] Hironori Takimoto, Tsubasa Kuwano, Yasue Mitsukura, Hironobu Fukai and Minoru Fukumi, "Appearance-age Feature Extraction from Facial Image Based on Age Perception", Tokyo, JAPAN, 2007, pp. 1-6

[6] Ramesha K, K B Raja, Venugopal K R and L M Patnaik, "Feature Extraction based Face Recognition, Gender and Age Classification", India, 2010, pp.1-10

[7] Özlem Özbudak, Mürvet Kırc1, Yüksel Çakır, Ece Olcay Güneş, "Effects of the Facial and Racial Features on Gender Classification", Turkey, 2010, pp. 1-4

\section{WEBSITES:}

[8] http://opencv.willowgarage.com/wiki/ FaceDetection

[9] http://libface.sourceforge.net/file/Home.html

[10] http://sourceforge.net/projects/faint/files/ 